PROTOPLANETARY DISKS

\section{Family portraits}

After more than five years of full Atacama Large Millimeter/submillimeter Array (ALMA) science operations, we have become used to seeing beautifully resolved images of the disks of gas and dust around young stars, and more importantly, clear signs of substructure within those disks. With the protoplanetary disk around TW Hydrae being one target of early ALMA Science Verification observations, and the spectacular multitude of rings around HL Tauri providing one of the first ALMA 'wow' moments, circumstellar disks have been a prime target to show off ALMA's impressive angular resolution. It is more enlightening and allows for more robust conclusions when galleries of protoplanetary disk portraits are presented together, and recently ALMA images of a dozen disks in Taurus have been published by Feng Long et al. (Astrophys. J. 869, 17; 2018) and twenty nearby disks have been imaged by the DSHARP collaboration (Sean M. Andrews et al. Astrophys. J. Lett., in the press; preprint available at http:// arxiv.org/abs/1812.04040) - pictured.

In the first of ten papers, Andrews and collaborators provide an overview of their twenty-disk study that is followed up in depth in the rest of the series. A key goal of DSHARP is to identify and characterize substructures in the distribution of dust within these disks, hence the name: Disk Substructures at High Angular Resolution Project. To do that, the team has tried to be as consistent as possible in observing their targets, obtaining data at the same angular scale, 35 milliarcseconds ( $\sim 5$ au at the distance of the sample) in the $240 \mathrm{GHz}$ (1.25 mm) continuum.

Some conclusions are immediately evident: all disks in the sample have substructure, even the youngest. In many cases this substructure is manifested as a set of bright, concentric rings around the central star, with dark gaps separating them. In other cases there are spirals and arcs, including in systems where the host stars are not thought to be multiples. Axisymmetry is very common, but not ubiquitous.

Other conclusions require modelling in order to unpick what is happening - particularly when it comes to

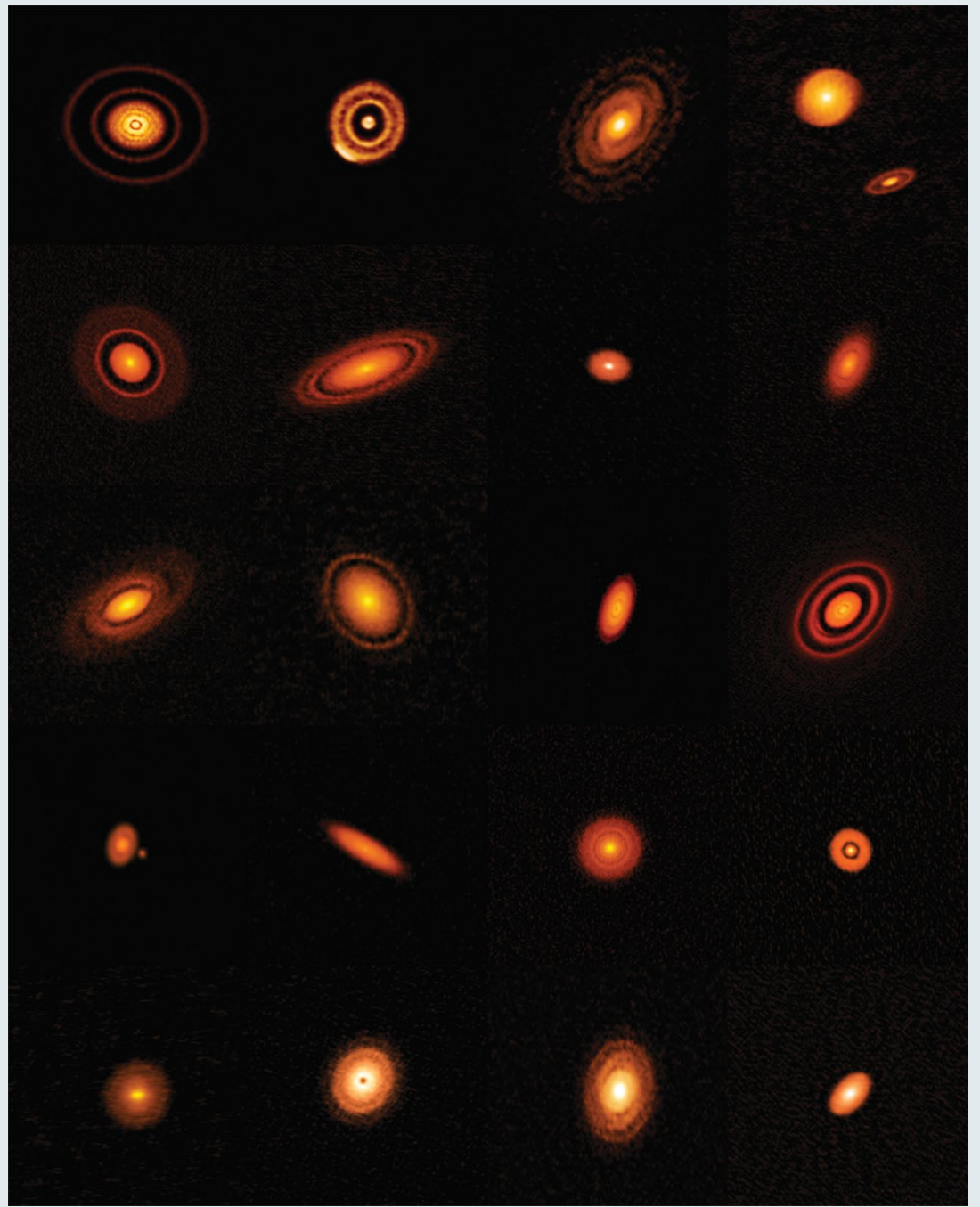

Credit: ALMA (ESO/NAOJ/NRAO), S. Andrews et al.; NRAO/AUI/NSF, S. Dagnello

understanding the underlying cause of the gaps in disks. Initial thoughts were that disk gaps were driven by the presence of planets; however, chemical modelling showed an association between the snowlines of various volatile molecules and gaps in molecular emission. Now that sample sizes are larger, the argument for planets has reasserted itself, with, for instance, the paper from Long et al. showing that there are discrepancies between gap locations and the expected location of ( $\mathrm{CO}$ and $\mathrm{N}_{2}$ ) snowlines. DSHARP papers VI and VII help to cement the relationship between solids and gaps, with the former paper showing that in models dust grains can become trapped in pressure bumps, leaving gaps, and the latter showing that hydrodynamics simulations of low-mass planets interacting with disks can reproduce observed patterns of rings and gaps.

\section{Paul Woods}

Published online: 7 February 2019

https://doi.org/10.1038/s41550-019-0691-5 